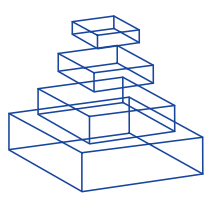

\title{
How does nature program neuron types?
}

\author{
Alexander Borst \\ Department of Systems and Computational Neurobiology, \\ Max-Planck-Institute of Neurobiology, Germany \\ Correspondence: borst@neuro.mpg.de
}

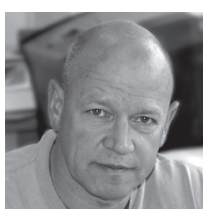

Alexander Borst obtained his $P h D$ on olfactory learning in Drosophila under the supervision of Martin Heisenberg from the University of Würzburg. In 1984, he took a position as a Research Scientist at the Max Planck Institute of Biological Cybernetics in Tübingen, Germany, switching from olfaction to the fly visual system. In 1993 he became Head of a Junior Research Group at the Friedrich-MiescherLaboratory, Tübingen. He was Professor at the UC Berkeley from 1999-2001. Since 2001, he is Director at the Max Planck Institute of Neurobiology, Martinsried/ Munich, Germany, and Head of the department of 'Systems and Computational Neurobiology'. His research focuses on computation in single neurons and small neural circuits. His lab combines electrophysiology, calcium imaging and genetic techniques with computational approaches.
Neurons come in different flavors. Once a recording is established, the electrophysiological properties, either in response to current injection or in response to sensory stimulation, are found to be characteristic for each neuron type, very much the same as their typical dendritic anatomy or axonal projection. In invertebrates, these statements even account for individual neurons occurring only once in each hemisphere of a ganglion. While these response characteristics have long been thought to be due to a typical composition of membrane currents, characteristic for each neuron type and constant across the different members of this type, this view has been challenged by a series of investigations performed on various neurons (beautifully reviewed in Marder and Goaillard, 2006). One of these studies was performed on the stomatogastric ganglion of the crab. This ganglion consists of only a handful of neurons, together forming two oscillatory networks one controlling the gastric mill and the other the pyloric rhythm of the animal. Each of the neurons can be named according to its invariant electrophysiological response properties: some of them are endogenously bursting, while others are passively following the rhythm of the others. Looking at the different membrane currents underlying the bursting behavior in the same neurons in different individuals, the surprising finding was that every neuron seemed to be bursting by a different mechanism: while in all of them the same basic set of membrane currents was present, their pattern of maximal ion conductances was found to widely differ in each case. Yet: all of them were bursting in an almost indistinguishable mode. Not too astonishingly, the amount of mRNA encoding for the membrane channels underlying the different currents was found to vary along with the maximum conductance values measured electrophysiologically (Schulz et al, 2006). Along similar lines, the work of Turrgiano, Nelson and colleagues (Turrigiano et al, 1998) has shown that synaptic strength is another parameter that is regulated such as to remain in an operating range appropriate to neuronal function.

What does this mean? Although never stated explicitly in this way, everyone in the field would probably think that a neuron's functional identity is the result of the expression of some sort of cell-specific transcription factor which in turn controls the expression of a set of ion channels in a cell-specific relative amount to each other. This way, the neuron would reveal its typical shape of action potential, its typical current-spike frequency rela- 
tionship and other characteristics like plateau potential or bursting mode. This would also be the way that every biophysical neuron-model is set up (e.g. Wicher et al, 2006) with the maximum conductances of all the different currents being defined in the parameter file executed upon initializing the simulation software. However, this does not seem to be the way that nature programs it. Instead, in real neurons, something else seems to be defined as characteristic for each neuron, and this is its overall behavior (its electrical "character"). From the studies mentioned above, one can conclude that neurons are given a goal like being 'bursting' and each neuron has to find a combination of maximum conductances that does the job. Once this point (the 'set point') is reached, the neuron is stable and keeps on expressing the ion channels in this fixed relative amount. When thinking about it in programming terms, this would be identical to parameter fitting in a model simulation: the task is to find the minimum of the error function between the desired output (the 'target') and the actual output of the model (e.g. Borst and Haag, 1996; Druckmann et al, 2007). This immediately poses a lot of questions: how on earth is the target function defined genetically? How is the actual output function measured by the neuron, and how is the error between target and the actual firing pattern measured? How is the minimum recognized? Obviously, no one today has an idea.

Most neuroscientists will readily agree that 'homeostasis' is at the heart of all biological phenomena. But, somehow it has been largely ignored in most of the studies in cellular neuroscience. On the other hand, the relevance of this design principle of a fixed and cell-specific set-point can hardly be overestimated. How else could it be that drugs prescribed to make up for the loss of transmitter in a locally restricted area of the brain are delivered systemically, affecting all receptors in all neurons that express them in the brain? Just think of what would happen if, in a network simulation with biophysically realistic neuron models, one altered the baseline activation of all GABA receptors by a factor of 5 or 10 ? Would the network still be functional? Of course it will not. But this is the difference between the real nervous system and the way we understand and model it at the moment: in the real nervous system, those neurons lacking GABAergic input due to e.g. neurodegeneration, will take the opportunity of the additional GABA to happily return to their set-point, while all others driven away from their set point by the medication, will find some means to homeostatically go back to their appropriate set-point after a while. Understanding these phenomena at the cellular level, thus, will lead not only to a deep understanding of how a neuronal identity is genetically programmed, it will also lead to an understanding of plasticity in a much more general way as the basic way that neurons are built and guaranteed to robustly perform in a largely unpredictable environment.

\section{REFERENCES}

Borst, A., Haag, J. (1996). The intrinsic electrophysiological characteristics of fly lobula plate tangential cells: I. Passive membrane properties. J. Computat. Neurosci. 3, 313-336.

Druckmann, S., Banitt, Y., Gidon, A., Schuermann, F., Markram, H., Segev, I. (2007). A novel multiple objective optimization framework for constraining conductance-based neuron models by experimental data. Frontiers in Neuroscience. 1, 7-18.

Marder, E., Goaillard, J-M. (2006). Variability, compensation and homeostasis in neuron and network function. Nature Review Neuroscience. 7, 563-574.
Schulz, D. J., Goaillard, J. M., Marder, E. (2006). Variable channel expression in identified single and electrically coupled neurons in different animals. Nature Neurosci. 9, 356-362.

Turrigiano, G. G., Leslie, K. R., Desai, N. S., Rutherford, L. C., Nelson, S. B. (1998). Activitydependent scaling of quantal amplitude in neocortical neurons. Nature. 391, 892-896.

Wicher, D., Berlau, J., Walther, C., Borst, A. (2006). Peptidergic counter-regulation of $\mathrm{Ca} 2+$ and $\mathrm{Na}+-$ dependent K-currents modulates the shape of action potentials in neurosecretory insect neurons. J. Neurophysiol. 95, 311-322.
Front. Neurosci. (2008) 2, 1: 4-5. doi: 10.3389/ neuro.01.016.2008

Copyright (c) 2008 Borst. This is an open-access publication subject to an exclusive license agreement between the authors and the Frontiers Research Foundation, which permits unrestricted use, distribution, and reproduction in any medium, provided the original authors and source are credited. 